\title{
Increased SLC4A11 expression is associated with poor prognosis of gastric cancer
}

\author{
Mengjing Sun ${ }^{1,2}$, Yue Qi ${ }^{3}$, Qin Jin ${ }^{4}$, Huijun Zhu ${ }^{4}$, Yao Wang ${ }^{5 *}$, Xiaohui Jiang ${ }^{6 *}$ \\ ${ }^{1}$ Department of Clinical Biobank, Affiliated Hospital of Nantong University, Nantong, Jiangsu, 226001, China; \\ ${ }^{2}$ Department of Pathology, Medical School of Nantong University, Nantong, Jiangsu, 226001, China; \\ ${ }^{3}$ Department of Oncology, Medical School of Nantong University, Nantong, Jiangsu, 226001, China; \\ ${ }^{4}$ Department of Pathology, Affiliated Hospital of Nantong University, Nantong, Jiangsu, 226001, China; \\ ${ }^{5}$ Department of General Surgery, Affiliated Hospital of Nantong University, Nantong, Jiangsu, 226001, China; \\ ${ }^{6}$ Department of General Surgery, Nantong Tumor Hospital, Nantong, Jiangsu, 226001, China.
}

\begin{abstract}
Solute carrier family 4 member 11 (SLC4A11) is a member of the SLC4 family of bicarbonate transporters, which have been reported to encode sodium-coupled bicarbonate transporters. In this study, SLC4A11 mRNA and protein expression in stomach tissue of 441 patients and 5 cell lines were detected by quantitative reverse transcription-polymerase chain reaction, Western blot, and immunohistochemistry. SLC4All expression was predictively analyzed using Oncomine online resources. The results revealed that SLC4A11 mRNA and protein levels were both significantly higher in gastric cancer than those in normal gastric tissues (both $P<0.001$ ). Statistical analysis revealed that SLC4A11 high expression was associated with Her-2 expression $(P=0.018), \mathrm{N}$ stage $(P=0.031)$, TNM stage $(P=0.015)$, and preoperative CEA levels $(P=0.017)$. In univariate and multivariate analyses, SLC4A11 overexpression and advanced $N$ stage showed a great prognostic value for 5-year survival. Our results suggest that SLC4A11 plays an important role in gastric carcinoma development and progression and that SLC4A1l could be a significant prognostic marker for gastric cancer patients.
\end{abstract}

Keywords: gastric cancer, SLC4A11, IHC, prognosis

\section{INTRODUCTION}

Gastric cancer (GC) is one of the most common infection-related malignancies and the third leading cause of cancer-associated deaths worldwide ${ }^{[1]}$. In China, the incidence and mortality of GC rank second and third, respectively ${ }^{[2]}$. Although gastroscopy has been an effective diagnostic method for $\mathrm{GC}^{[3]}$, therapies including surgical resection and chemotherapy have only added modest benefits to patient outcomes ${ }^{[4]}$. Due to these limitations, numerous studies have been focused on molecular-targeted therapy for GC and other cancers ${ }^{[5,6]}$. Given the high prevalence of gastric tumorigenesis and the high recurrence rate of stomach cancer, there is an urgent need to find effective prognostic biomarkers that can be used to evaluate each patient's chances of survival.

Previous studies have shown that the regulation of intracellular and extracellular $\mathrm{pH}$ by the primary

*Correspondence to: Yao Wang, Department of General Surgery, Affiliated Hospital of Nantong University, Nantong, Jiangsu, 226001, China. Email: wangyao@ntu.edu.cn; Xiaohui Jiang, Department of General Surgery, Nantong Tumor Hospital, Nantong, Jiangsu, 226001, China. E-mail: jxhyj1@163.com.

The authors have no conflicts of interest. 
transportation of $\mathrm{HCO}_{3}{ }^{-}$impacts cell metabolism and growth ${ }^{[7]}$. The solute carrier family 4(SLC4) of $\mathrm{HCO}_{3}{ }^{-}$ transporters plays a pivotal role in this regulation, as these proteins can move $\mathrm{HCO}_{3}{ }^{-}$into or out of cells ${ }^{[8]}$. Solute carrier family 4 member 11 (SLC4A11) is a member of the SLC4 family of bicarbonate transporters that has been reported to encode a transmembrane protein called BTR1 in corneal endothelial cells ${ }^{[9-11]}$. SLC4All is enriched in the corneal endothelium and multiple renal epithelia ${ }^{[12]}$. Numerous studies have indicated that SLC4All mutations can induce some symptoms of blinding corneal dystrophies, Fuchs endothelial corneal dystrophy (FECD), and congenital hereditary endothelial dystrophy (CHED $)^{[13,14]}$. Additionally, a recent study that used dataset analysis showed that high SLC4A1l expression is a substantive predictor of poor overall survival in ovarian cancer $^{[15]}$. However, research into the relationship between SLC4A11 and clinical outcomes of GC has not been performed.

Here, we discovered an enrichment of SLC4All in GC cells after examining SLC4All expression in gastric tumors by quantitative reverse transcriptionpolymerase chain reaction (qRT-PCR), Western blot, and immunohistochemistry (IHC). Meanwhile, we further conducted an analysis of SLC4A1l expression in relationship to patients' clinical characteristics and prognosis. Finally, databases were used to confirm SLC4A11 expression in GC using bioinformatics analysis.

\section{MATERIALS AND METHODS}

\section{Bioinformatics analysis}

In this study, we used Oncomine (https://www. oncomine.org), an online database of RNA and DNA sequencing information rooted in the Cancer Genome Atlas to perform data collection. "SLC4All" and "Gastric Cancer" were input as the search terms; at the same time, a minimum threshold of a 1.5-fold change with a $P$-value of 0.05 was set for data acquisition.

\section{Tissue specimens and patient clinical data}

Formalin-fixed, paraffin-embedded tissues were obtained from surgical cases stored in the Clinical Biobank of the Affiliated Hospital of Nantong University from 2004 to 2009 . In total, 543 stomach tissue samples were collected from 441 patients, including 281 GCs (15 intestinal type adenocarcinomas, 131 diffuse type adenocarcinomas, 117 mixed type adenocarcinomas, one small cell carcinoma, one carcinoid, one undifferentiated carcinoma, one adeno squamous carcinoma, 3 squamous cell carcinomas, and 11 mucinous adenocarcinomas), 70 matched normal surgical margins (MNSM), 21 chronic gastritis (CG), 20 intestinal metaplasia (IM), 28 lowgrade intraepithelial neoplasias (LIN), and 21 highgrade intraepithelial neoplasias (HIN). Clinical data were gathered from patient records. No patients had received chemotherapy, radiotherapy, or immunotherapy before surgery. Patient ages ranged from 19 to 87 years old. The 5 -year survival rates were counted from the first day after surgery to the date of death or final follow-up.

A tissue microarray (TMA) was prepared as previously reported ${ }^{[16]}$ by the Tissue Microarray System (Quick-Ray, UT06, UNITMA, Seoul, South Korea) in the Clinical Biobank Department of Affiliated Hospital of Nantong University. Patients were classified according to medical records by sex, histological type, and TNM stage. The research protocol was approved by the local hospital's Ethics Committee, and the study was conducted according to the guidelines of the Affiliated Hospital of Nantong University.

\section{qRT-PCR}

Fresh GC ( $n=28)$ and MNSM ( $n=28)$ samples were obtained from the Clinical Biobank of the Affiliated Hospital of Nantong University for qRT-PCR analysis. TRIzol reagent (Invitrogen, Carlsbad, CA, USA) was used to extract total RNA from frozen tissues. The extracted RNA was then converted to cDNA using a High Capacity RNA-to-cDNA Kit (4387406; Life, USA). qRT-PCR was performed using an ABI 7500 system with a SYBR Green Master Mix (TaKaRa, Dalian, China). The primers for SLC4A1l amplification were as follows: forward 5'-CAATGACGAGAACACAGA-3', and reverse 5'-TCAGCAGAATCACCAATG-3'(Invitrogen, Shanghai, China). Glyceraldehyde-3-phosphate dehydrogenase (GAPDH) was amplified as an internal control (forward 5'-CATGGTCTACATGTTCCAGT-3' and reverse 5'-GGCTAAGCAGTTGGTGGTGC-3'). The amplification conditions were $30 \mathrm{~min}$ at $42^{\circ} \mathrm{C}$ for reverse transcription, $10 \mathrm{~min}$ at $95{ }^{\circ} \mathrm{C}$ for initial denaturation and Taq activation, and then by 35 cycles at $95^{\circ} \mathrm{C}$ for $15 \mathrm{~s}, 60{ }^{\circ} \mathrm{C}$ for $1 \mathrm{~min}$. Target mRNA levels were determined by standard curve method and expressed as arbitrary units. The experiment was performed in triplicate.

\section{Cell lines and cell culture}

One normal gastric mucosal epithelial cell line (ges1) and four gastric carcinoma cell lines (hgc27, mknl, mkn45, and snu719) were acquired from the Cobioer Biotechnology Company (Nanjing, China). All cells 
were cultured in RPMI-1640 (Gibco, Waltham, MA, USA) supplemented with $10 \%$ fetal bovine serum (Gibco) in a $37^{\circ} \mathrm{C}$ incubator with $5.0 \% \mathrm{CO}_{2}$.

\section{Western blot}

The method for Western blotting was as previously described ${ }^{[17]}$. The five cell lines were first digested with $0.25 \%$ Trypsin-EDTA (Gibco). To collect total cellular protein, cells were lysed in RIPA lysis buffer (plus PMSF) for 20 min on ice, six GC and six MNSM tissue specimens were homogenized with tissue grinder in lysis buffer. Then the mixture was collected and centrifuged at $12,000 \mathrm{~g}$, for $15 \mathrm{~min}$ at $4{ }^{\circ} \mathrm{C}$. The protein samples were separated by $10 \%$ SDSPAGE and transferred onto a polyvinylidene fluoride membrane. Following blocking with $5 \%$ skimmed milk powder for $2 \mathrm{~h}$ at room temperature, the membrane was incubated with rabbit anti-SLC4All antibody (1:200; \#PA5-53730; Thermo Fisher Scientific, Waltham, MA, USA) and mouse anti-GAPDH antibody (1:1,000; AB-M-M001; GOODHERE, Hangzhou, China) at $4{ }^{\circ} \mathrm{C}$ overnight. The next day, corresponding rabbit and mouse secondary antibodies (1:2,000; Abcam, Cambridge, UK) were incubated with the membrane, which was then scanned and detected with an ECL imager (Beyotime Institute of Biotechnology, Haimen, China) after repeated washing with Tris-buffered saline plus $0.2 \%$ Tween-20. Subsequently, immunoreactive bands were displayed and the grayscale of these bands were analyzed with Image J software (National Institutes of Health, Mary land, USA) ${ }^{[18]}$.

\section{IHC}

IHC staining for SLC4A11 protein in all included patients was performed on the TMA as follows ${ }^{[38,39]}$. Deparaffinized $4-\mu \mathrm{m}$ sections were dewaxed with xylene and rehydrated using a graded alcohol series $(100 \%, 95 \%$, and $70 \%)$ after drying. Before incubating with primary rabbit anti-SLC4All antibody (1:200; HPA018120; Atlas antibodies, Sweden) overnight at $4{ }^{\circ} \mathrm{C}$, endogenous peroxidase activity was quenched with $\mathrm{H}_{2} \mathrm{O}_{2}$ for 10 min, followed by antigen retrieval in citrate buffer. After washing with PBS, the slides were incubated with conjugated goat anti-rabbit IgG antibody (1:1,000; A2100; Abbkine, Inc., Redlands, CA, USA) for $30 \mathrm{~min}$, and then washed. Color was presented with an Elivision Plus DAB kit (Maixin, Fuzhou, China). All slides were short-lived counterstained with Gill's hematoxylin.

Stained TMA slides were observed and scored using a Vectra 3.0 Automated Quantitative Pathology Imaging System (PerkinElmer, USA). All scoring data were then verified by a pathologist without clinical information. As previously described ${ }^{[19,20]}$, the staining intensity was scored as: 0 (negative staining), 1 (weak staining), 2 (medium staining), and 3 (strong staining). The product of the percentage of stained cells and the intensity score was used as the final staining score and ranged from 0 to 300 . The cutoff point of the SLC4All expression score was statistically significant by X-tile software (Rimm Laboratory, Yale University; http://www.tissuearray.org/rimmlab). Here, 133 was chosen as the optimal cutoff point for SLC4A11 in GC based on the maximum $\chi^{2}$ value and estimated by log-rank test statistics with regard to overall survival (OS). Scores from 0 to 132 were regarded as low or no expression, while scores from 133 to 300 were regarded as high expression.

\section{Statistical analysis}

Statistical analyses were performed with SPSS 23.0 software (IBM Corp., Armonk, NY, USA). The correlations between SLC4A1l expression and clinicopathological factors were compared using the Student's $t$-test or Pearson's $\chi^{2}$ test. Kaplan-Meier survival analysis was used to estimate the survival rate of GC patients, which were then further analyzed by the log-rank test according to SLC4All expression. Univariate and multivariate Cox regression were used to investigate the prognostic value of SLC4All expression in GC. Student $t$-test was used to analyze qPCR and GC tissue Western bolt assay data, while a one-way ANOVA was used to evaluate differences in IHC and Western blot assays using GraphPad Prism 7 software (GraphPad Software, Inc., San Diego, CA, USA). A $P$-value of $<0.05$ was considered statistically significant for all tests.

\section{RESULTS}

\section{SLC4A11 expression analysis in the databases}

To confirm the results' reliability, bioinformatics analysis using the Oncomine database was performed. Consistent with the qRT-PCR results, SLC4A1l mRNA was found to be overexpressed in GC tissues compared with that in normal tissues (Fig. 1).

\section{SLC4A11 mRNA levels in GC and MNSM tissues}

To investigate SLC4All mRNA expression in GC patient tissues, total RNA was extracted from $28 \mathrm{GC}$ and 28 MNSM tissues and analyzed by qRT-PCR. This analysis showed that SLC4All mRNA expression was significantly higher in GC tissues than that in MNSM tissues $(2.201 \pm 0.222$ vs. $0.710 \pm 0.114$, $P<0.001$, Fig. 2). 
Comparison of SLC4A11 Across 6 Analyses

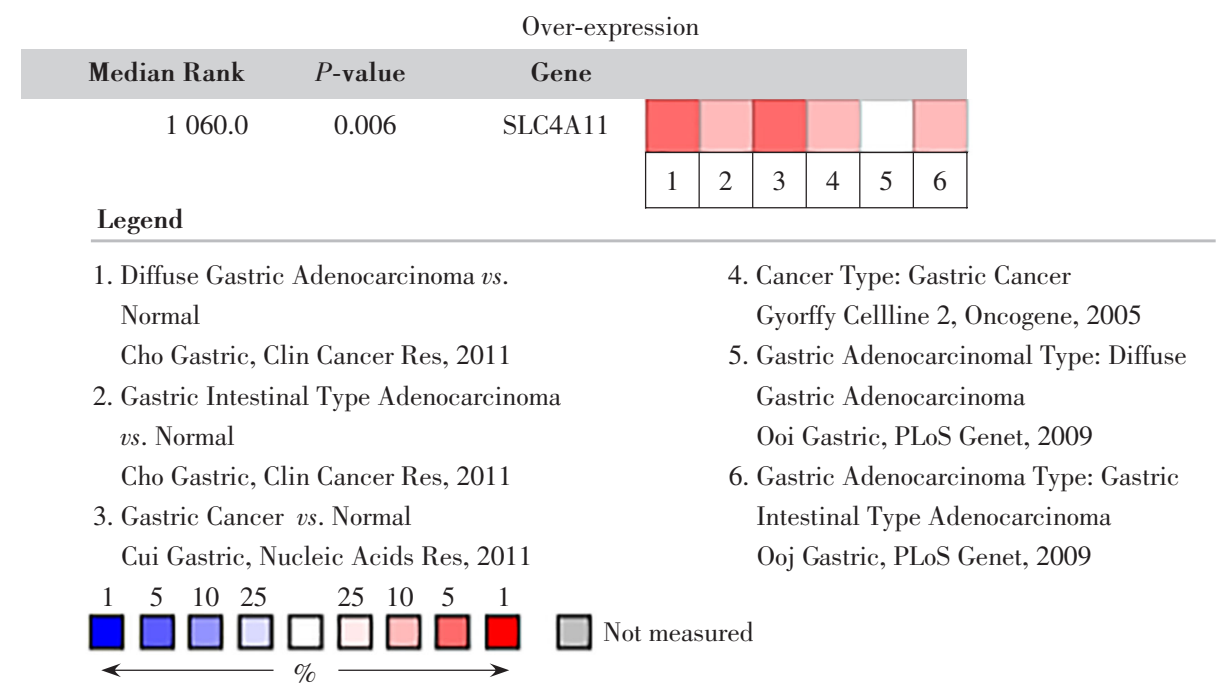

The rank for a gene is the median rank for that gene across each of the analyses.

The $P$-value for a gene is its $P$-value for the median-ranked analysis.

Fig. 1 SLC4A11 mRNA levels in the Oncomine database. mRNA expression of SLC4A11 in gastric cancer and normal tissues were analyzed by Oncomine. The intensity of color represents the respective SLC4A11 levels.

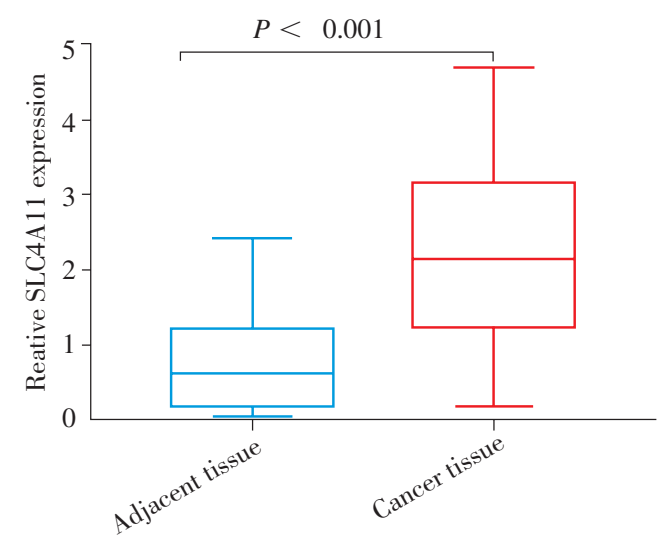

Fig. 2 SLC4A11 mRNA levels were significantly higher in cancerous tissues than that in normal tissues. SLC4A11 mRNA was detected by qRT-PCR and measured by quantitative analysis, $n=28$.

\section{Evaluating SLC4A11 protein expression in GC and normal gastric tissues}

Western blot analysis was used to measure SLC4All protein expression in five cell lines, six GC and six MNSM tissues. The results showed that SLC4All had decreased expression in ges-1 cells compared with those in all GC cell lines (Fig. 3), and higher expression in GC than those in in MSNM tissues (Fig. 4). These results agreed with the mRNA expression data from the qRT-PCR analysis.

To further confirm these findings, IHC was performed on the TMA to examine SLC4A11 expression in GC patients. As shown in Table 1, the high SLC4All expression rates were $14.29 \%$ in CG,

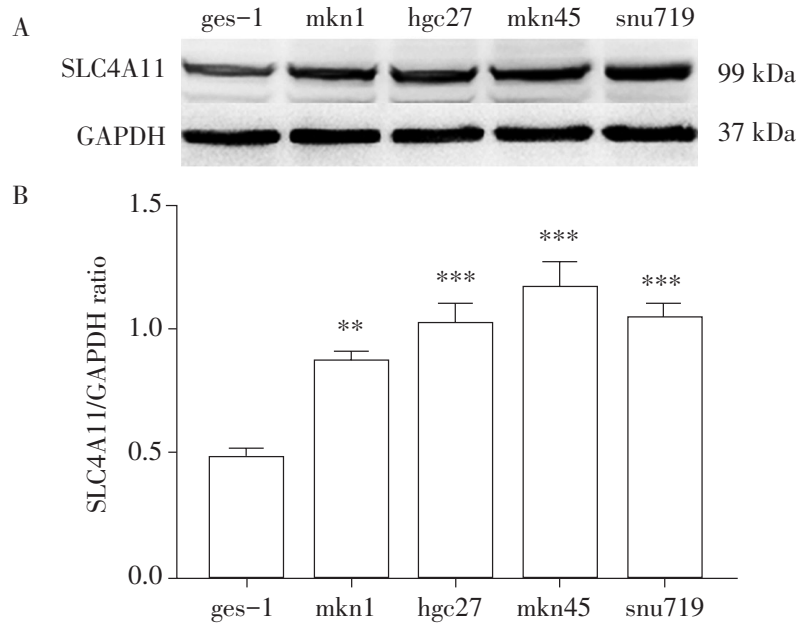

Fig. 3 Western blot analysis of SLC4A11 protein in ges-1, mkn1, hgc 27, mkn45, and snu719 cells. A: representative Western blots; B: quantitative analysis (ImageJ, NIH). Statistical significance was measured using one-way ANOVA, ${ }^{*} P<0.01,{ }^{* * *} P<0.001$ vs. ges $-1, n=3$.

$35.00 \%$ in IM, $32.14 \%$ in LIN, $28.57 \%$ in $\mathrm{HIN}$, $27.14 \%$ in MNSM, and $65.48 \%$ in GC, respectively. SLC4A11 expression was also increased with increasing malignancy grade $\left(\chi^{2}=61.068, P<0.001\right)$. Together, these findings indicated that SLC4All expression was significantly higher in GC than that in normal tissues, which was concordant with the qRT-PCR and Western blot analyses. Representative immunohistochemical staining for SLC4A1l is shown in Fig. 5 and Fig. 6. SLC4A11 expression H-score in different histopathological classification is given in Fig. 7 . 


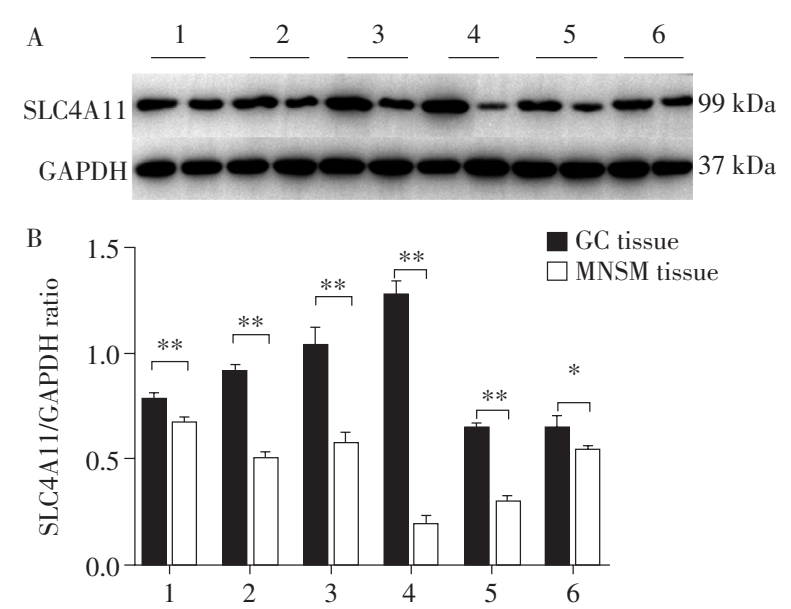

Fig. 4 Western blot analysis of SLC4A11 protein in GC and MNSM tissues. A: representative Western blot; B: quantitative analysis (ImageJ, NIH). Statistical significance was measured using $t$ test, ${ }^{*} P<0.05,{ }^{* *} P<0.01, n=3$.

\section{Association of SLC4A11 expression with clin- ical parameters in GC patients}

The correlations between SLC4A1l expression and clinicopathological factors were displayed in Table 2. The results revealed that SLC4All expression in GC was significantly associated with Her-2 status $\chi^{2}=8.043$, $P=0.018), \mathrm{N}$ stage $\left(\chi^{2}=8.875, P=0.031\right)$, TNM stage $\left(\chi^{2}=12.273, P=0.015\right)$, and preoperative carcinoembryonic antigen(CEA) level $\left(\chi^{2}=5.687, P=0.017\right)$. In contrast, there were no significant correlations between SLC4A1l expression and other clinical characteristics, including gender, age, differentiation, $\mathrm{T}$ stage, and preoperative CA19-9 level.

\section{High SLC4A11 expression predicts poor prognosis}

Univariate and multivariate analyses were used to

Table 1 SLC4A11 expression in stomach benign and malignant tissues

\begin{tabular}{|c|c|c|c|c|c|}
\hline Characteristic & Cases & Low or no expression & High expression & Pearson $\chi^{2}$ & $P$ \\
\hline & & & & 61.068 & $<0.001$ \\
\hline Intestinal metaplasia & 20 & $13(65.00)$ & $7(35.00)$ & & \\
\hline Low-grade intraepithelial neoplasia & 28 & $19(67.86)$ & $9(32.14)$ & & \\
\hline High-grade intraepithelial neoplasia & 21 & $15(71.43)$ & $6(28.57)$ & & \\
\hline Gastric cancer & 281 & $97(34.52)$ & $184(65.48)$ & & \\
\hline Surgical margin* & 70 & $51(72.86)$ & $19(27.14)$ & & \\
\hline
\end{tabular}

*: Epithelium without intestinal metaplasia or intraepithelial neoplasia from stomach cancer patients.

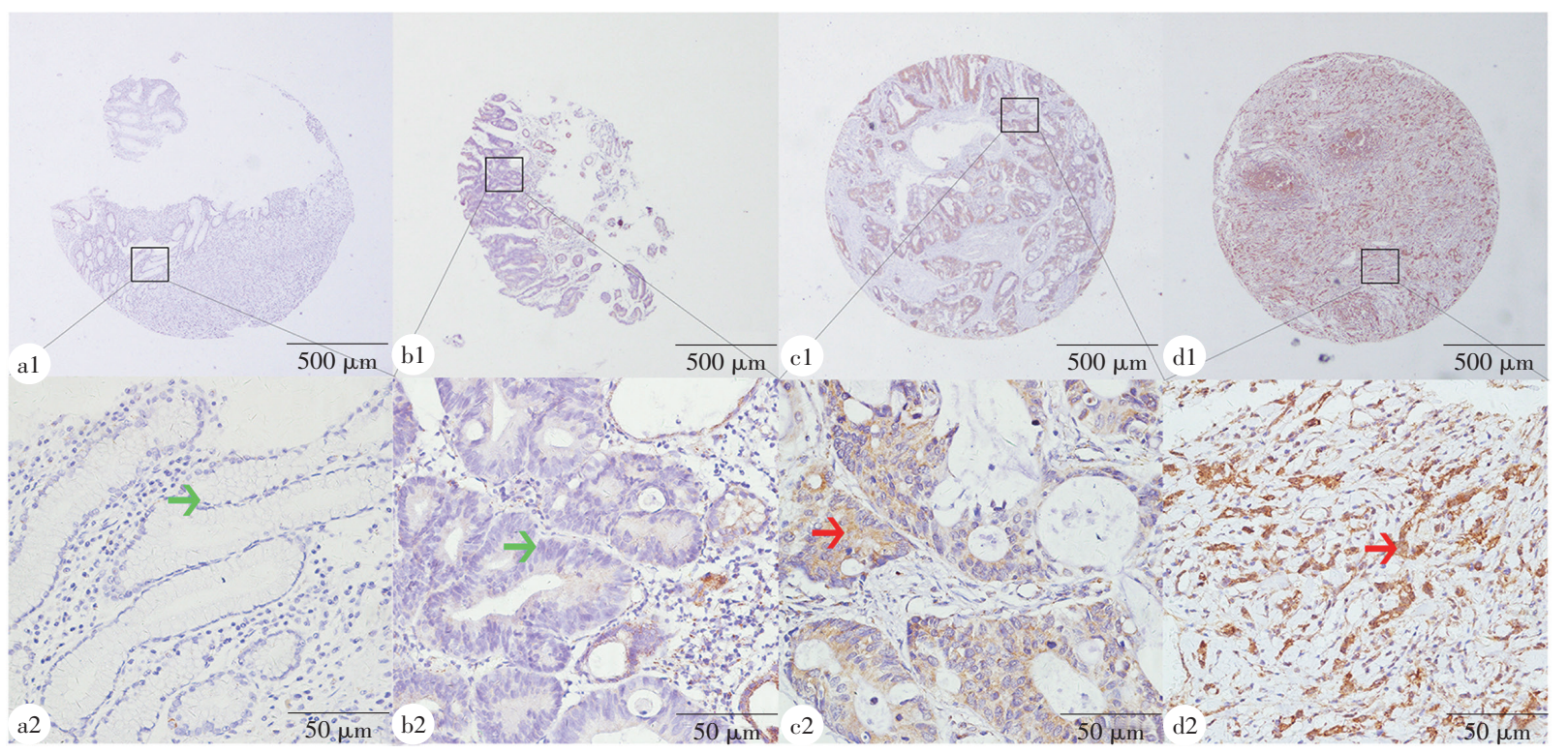

Fig. 5 Representative images of SLC4A11 protein expression in gastric carcinoma and non-tumor tissues from the TMA by immunohistochemistry. a1, a2: negative SLC4A11 expression in normal stomach mucosal epithelial tissue; b1, b2: weakly positive SLC4A11 expression in stomach intestinal metaplasia tissue; c1, c2: medium positive SLC4A11 expression in well-differentiated GC tissue; d1, d2: strongly positive SLC4A11 expression in poorly-differentiated GC tissue. Red arrows indicate positive SLC4A11 protein expression and green arrows show negative or weak SLC4A11 protein expression. Original magnification $40 \times($ bar $=500 \mu \mathrm{m})$ in the top row; original magnification $400 \times($ bar $=50 \mu \mathrm{m})$ in the bottom row. 


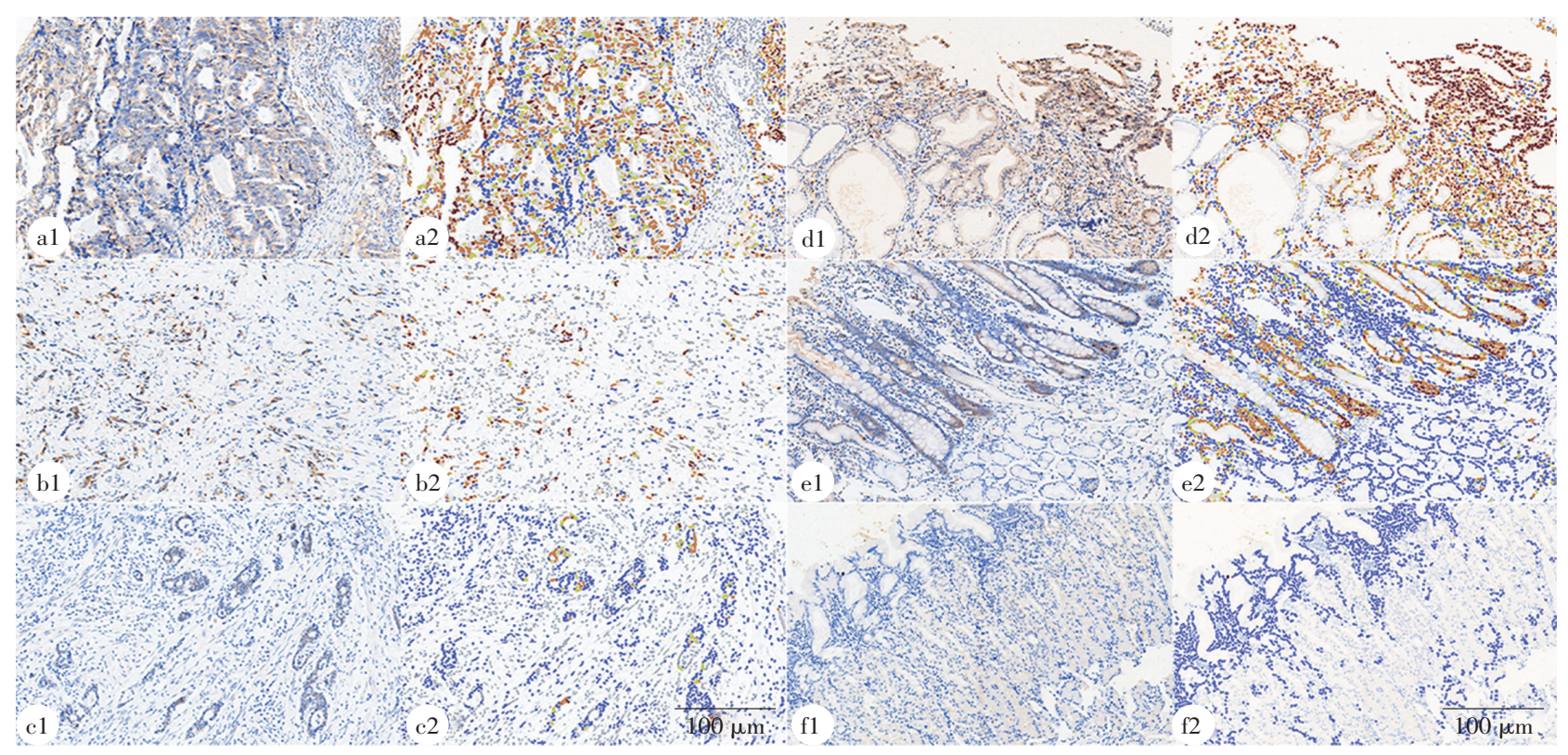

Fig. 6 Representative images of SLC4A11 protein expression in benign and malignant tissues from the TMA. a: Intestinal type adenocarcinoma with positive SLC4A11 protein expression $(\mathrm{H}$-score=166). b: Diffuse type adenocarcinoma with low positive SLC4A11 protein expression $(\mathrm{H}$-score=43). c: Mixed type adenocarcinoma with positive SLC4A11 protein expression $(\mathrm{H}$-score $=22)$. d: Intraepithelial neoplasia with positive SLC4A11 protein expression $(\mathrm{H}$-score=218). e: Intestinal metaplasia with low positive SLC4A11 protein expression $(\mathrm{H}$-score=78). f: Chronic gastritis with negative SLC4A11 protein expression $(\mathrm{H}$-score=0). 1: Images from immunohistochemical staining analysis. 2: Different positive intensity indicators for analysis of scanned images using Vectra 3.0 Automated Quantitative Pathology Imaging System (brown 3+, orange 2+, yellow 1+, blue 0)(bsr=100 $\mu \mathrm{m})$.

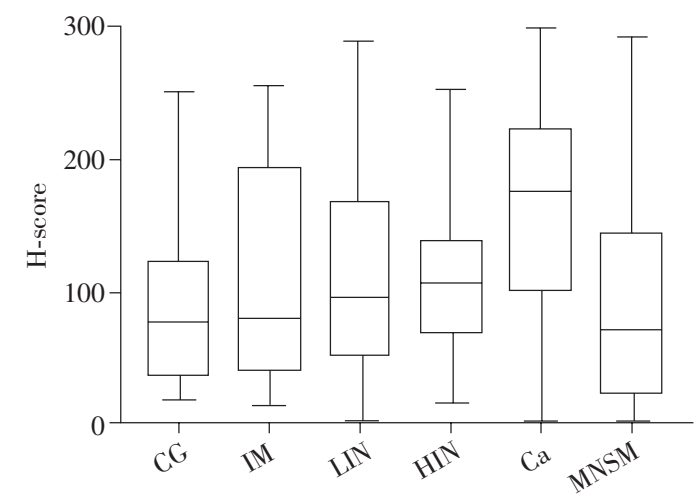

Fig. 7 SLC4A11 protein levels in benign and malignant tissues. Chronic gastritis (CG), intestinal metaplasia (IM), low-grade intraepithelial neoplasia (LIN), high-grade intraepithelial neoplasia (HIN), gastric cancer (GC) and matched normal surgical margins (MNSM) (software: GraphPad Prism 7).

investigate the prognostic value of SLC4A1l expression in GC (Table 3). Univariate analysis revealed that high SLC4A1l expression was associated with poor survival, along with other prognostic indicators including TNM stage (hazard ratio $[\mathrm{HR}]=1.528,95 \%$ confidence interval [CI]: $0.362-1.715, P<0.001)$, T stage $(\mathrm{HR}=1.719,95 \% \mathrm{CI}: 1.372-2.155, P<0.001), \mathrm{N}$ stage $(\mathrm{HR}=1.428,95 \% \mathrm{CI}: 1.259-1.619, P<0.001)$, preoperative CA19-9 level $(\mathrm{HR}=1.938,95 \% \mathrm{CI}$ : 1.196-3.141, $P=0.007$ ), and preoperative CEA level $(\mathrm{HR}=1.792,95 \% \mathrm{CI}: 1.180-2.722, P=0.006)$. Next, the significant factors from the univariate analysis were brought into a multivariate Cox regression model, which showed that SLC4All expression $(\mathrm{HR}=1.439,95 \% \mathrm{CI}: 1.043-1.984, P=0.027)$ and $\mathrm{N}$ stage $(\mathrm{HR}=1.675,95 \% \mathrm{CI}: 1.335-2.100, P<0.001)$ were independent indicators of an unfavorable prognosis (Fig. 8).

\section{DISCUSSION}

In this study, the results revealed that SLC4A11 expression was higher in GC tissues compared with that in MNSM tissues. Furthermore, we discovered that high SLC4A11 levels were significantly correlated with Her-2 expression, advanced N stage, advanced TNM stage, and elevated preoperative CEA levels. Additionally, high SLC4A1l expression was positively associated with poor survival in GC patients.

SLC4A11 is a member of the SLC4 family, which contains the products of 10 genes that are also known to be bicarbonate transporters. These gene products play a crucial role in maintaining efficient $\mathrm{pH}$ regulation $^{[21,22]}$. The SLC4 gene family consists of three major functional groups, which include $\mathrm{Na}^{+}$-independent $\mathrm{Cl}^{-} / \mathrm{HCO}_{3}{ }^{-}$exchangers (SLC4Al-3), $\mathrm{Na}^{+}$-coupled $\mathrm{HCO}_{3}{ }^{-}$transporters (SLC4A4, $-4 \mathrm{~A} 5,-4 \mathrm{~A} 7,-4 \mathrm{~A} 10$ ), and $\mathrm{Na}^{+}$-driven $\mathrm{Cl}^{-} / \mathrm{HCO}_{3}{ }^{-}$exchangers (SLC4A8) ${ }^{[23]}$. The molecular actions of SCL4A9 and SCL4A11 remain controversial. As the most divergent gene of the SLC4 
Table 2 Relationship between the expression of SLC4A11 and clinicopathological characteristics in gastric cancer patients

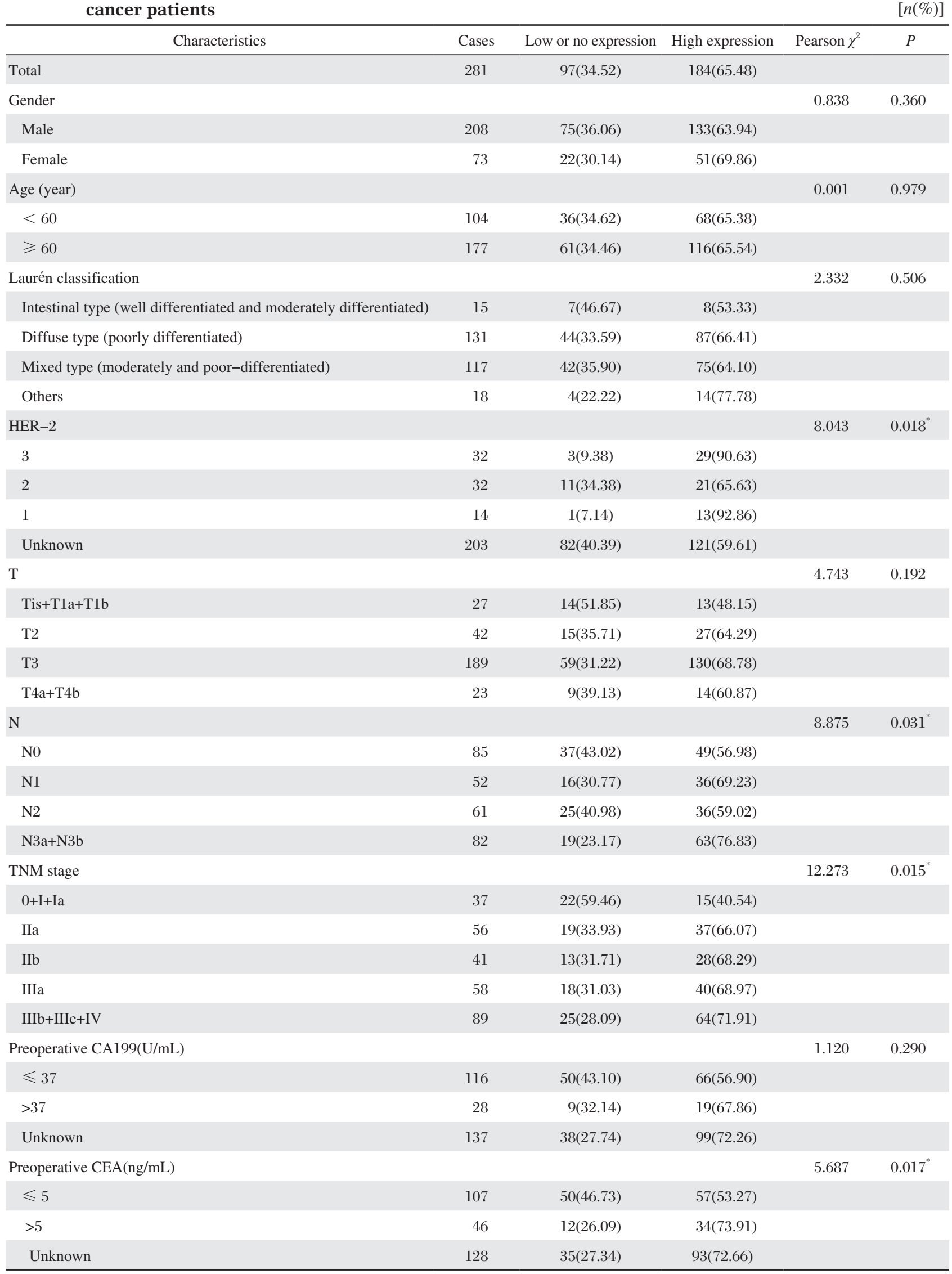

Others: small cell carcinoma, one case; carcinoid, one case; undifferentiated carcinoma, one case; adenosquamous carcinoma, one cases; squamous cell carcinoma, 3 cases; mucinous adenocarcinoma, 11 cases; ${ }^{*} P<0.05$. 
Table 3 Univariate and multivariat analysis of prognostic factors for 5-year survival in gastric cancer

\begin{tabular}{|c|c|c|c|c|c|c|}
\hline \multirow{2}{*}{ Characteristics } & \multicolumn{3}{|c|}{ Univariate analysis } & \multicolumn{3}{|c|}{ Multivariate analysis (adjusted for age) } \\
\hline & HR & $P>|z|$ & $95 \% \mathrm{CI}$ & HR & $P>|z|$ & $95 \% \mathrm{CI}$ \\
\hline SLC4A11 expression (High vs. low and none) & 1.563 & 0.006 & $1.135 \sim 2.152$ & 1.439 & 0.027 & $1.043 \sim 1.984$ \\
\hline Age (years, $\leqslant 60 v s .>60$ ) & 1.272 & 0.123 & $0.937 \sim 1.728$ & & & \\
\hline Gender (Male vs. Female) & 1.007 & 0.965 & $0.725 \sim 1.400$ & & & \\
\hline Laurén classification (Intestinal type $v s$. Diffuse type $v s$. Mixed type) & 1.071 & 0.513 & $0.871 \sim 1.318$ & & & \\
\hline Her-2 (1 vs. 2 vs. 3$)$ & 0.895 & 0.397 & $0.605 \sim 1.221$ & & & \\
\hline TNM stage ( 0 and Ia and Ib vs. IIa vs. IIlb vs. IIIa vs. IIIb and IIIc and IV) & 1.528 & $<0.001$ & $1.362 \sim 1.715$ & & & \\
\hline $\mathrm{T}$ (Tis and $1 \mathrm{a}$ and $1 \mathrm{~b}$ vs. 2 vs. 3 vs. $4 \mathrm{a}$ and $4 \mathrm{~b})$ & 1.719 & $<0.001$ & $1.372 \sim 2.155$ & & & \\
\hline $\mathrm{N}(0 v s .1$ vs. $2 v s .3 \mathrm{a}$ and $3 \mathrm{~b})$ & 1.428 & $<0.001$ & $1.259 \sim 1.619$ & 1.675 & $<0.001$ & $1.335 \sim 2.100$ \\
\hline Preoperative CA199 (U/mL, $\leqslant 37 v s .>37)$ & 1.938 & 0.007 & $1.196 \sim 3.141$ & & & \\
\hline Preoperative CEA (ng/mL, $\leqslant 5 v s .>5)$ & 1.792 & 0.006 & $1.180 \sim 2.722$ & & & \\
\hline
\end{tabular}
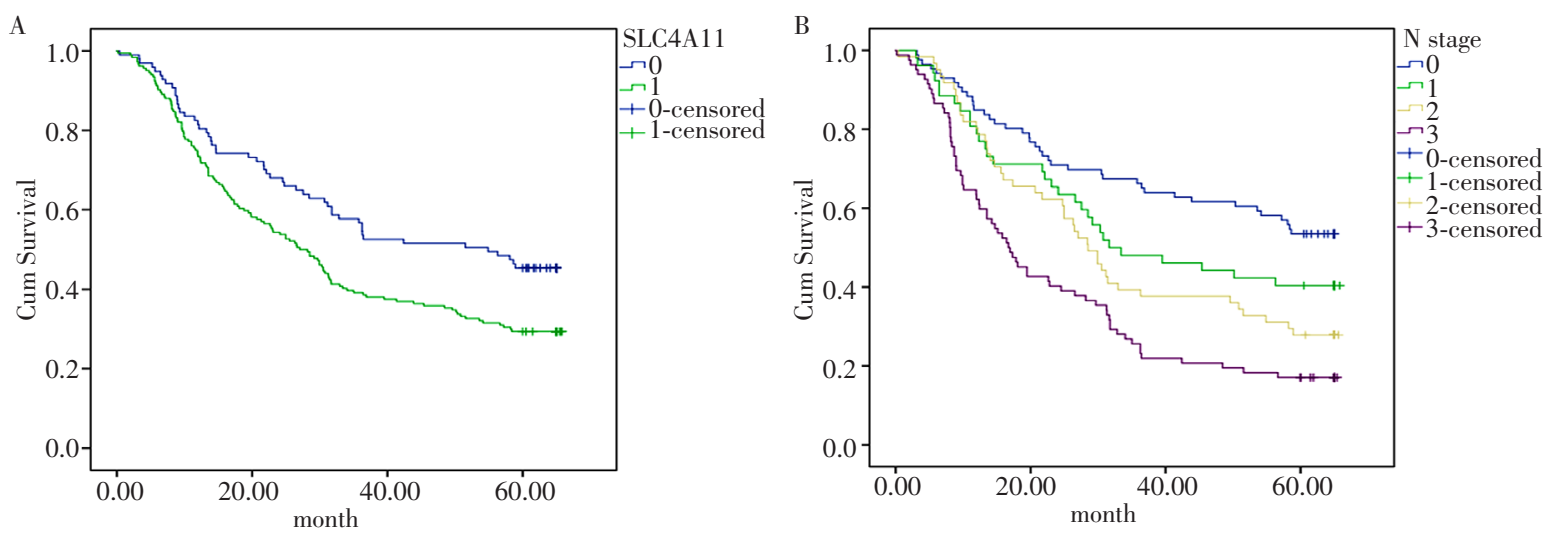

Fig. 8 Survival curves for gastric carcinoma by the log-rank test and Kaplan-Meier method. A: overall survival curves of SLC4A11 ${ }^{+}(1$, green) and SLC4A11 $(0$, blue). B: overall survival curves by tumor stage, N0 (blue, 0), N1 (green, 1), N2 (light brown, 2), N3a+3b (purple, 3).

family, SCL4A11 shares the lowest identity in aminoacid sequence among all family members, and recent research has indicated it as a $\mathrm{Na}^{+} / \mathrm{OH}^{-}$transporter rather than a $\mathrm{HCO}_{3}{ }^{-}$transporter ${ }^{[21,24]}$. SLC4 proteins play important roles in regulating acid-base homeostasis, such as the transport of $\mathrm{CO}_{2}$ by erythrocytes, the carriage of $\mathrm{H}^{+}$or $\mathrm{HCO}_{3}{ }^{-}$by various epithelia, the control of cell volume, and the regulation of intracellular $\mathrm{pH}^{[21]}$. SLC4A11 is a membrane of the SLC4 family of transport proteins that allows $\mathrm{Na}^{+}, \mathrm{H}^{+}, \mathrm{OH}^{-}, \mathrm{NH}_{4}^{+}$, bicarbonate, and borate to permeate the cell ${ }^{[9]}$. A novel nonsense mutation, as well as insertions and deletions ${ }^{[25]}$ in SLC4A1l have been repeatedly reported and studied, as these lead to congenital hereditary endothelial dystrophy (CHED) ${ }^{[26-28]}$. These mutations do not directly induce cell death in an in vitro model $^{[29]}$, rather the loss of SLC4All protein causes an infiltration imbalance, which induces oxidative and mitochondrial damage that causes corneal endothelial cells to die ${ }^{[30]}$. Very recently, there was a promising study that showed a meaningful connection between SLC4A1l deficiency and a reduction in nuclear fac- tor erythroid 2-related factor 2 (NRF2) expressions over the course of CHED and Fuchs endothelial corneal dystrophy(FECD) by increasing levels of reactive oxygen species. Compared with control siRNAtransfected cells, p53 and Bax (pro-apoptotic gene) were increased in SLC4A1l knockdown cells ${ }^{[31]}$. Other research has indicated that SLC4All knockdown can reduce cell proliferation and induce apoptosis by upregulating apoptotic genes or activating the apoptosome, which comprises cytochrome $\mathrm{C}$ and apoptotic protease-activating factor 1 (Apaf-1 $)^{[32]}$.

SLC4A11 is an important paralog of SLC4A. SLC4A has been reported to reduce tumor cell growth when disrupted by reducing the expression of carbonic anhydrase IX (CA9) ${ }^{[33]}$. In colon and breast cancer, SLC4A4 contributes to $\mathrm{HCO}_{3}{ }^{-}$transport and tumor cell phenotypes ${ }^{[34]}$. SLC4A1l consists of a twodomain structure: a $41 \mathrm{kDa}$-terminal cytoplasmic domain $(\mathrm{CD})$ and a $57 \mathrm{kDa}$ integral membrane domain (MD), which lead to SLC4All staining being observed in the cytoplasm and membrane ${ }^{[35]}$. The structure of CD-SLC4A1l is similar to that of the SLC4Al 
CD by homology modeling ${ }^{[36]}$. Finally, SLC4Al was observed to be enriched in renal cell carcinoma ${ }^{[37]}$. These findings support the possibility of SLC4A11 cancer-related functions. Taken together, SLC4A11 may play a significant role in promoting the growth of GC cells, and is associated with a poor prognosis of GC patients.

Further research is warranted to determine whether SLC4A11 is correlated with some anticancer drugs. Specific drugs could be applied to GC patients at high levels to decrease its expression, whether it can inhibit the growth of GC cells and the remedial effects requires verification by follow-up studies.

There were some limitations in our research. First, because this was a retrospective study, the results do not represent other GC cases. We will need to expand the research population for a prospective study. Second, the mechanism through which SLC4A1l influences the development and progression of GC has not been investigated. More mechanistic studies should be devoted to understanding the biological roles of the SLC4All expression in GC.

In summary, this study suggests that SLC4All expression might serve as an independent factor to evaluate the prognosis of GC patients. Our results represent an advancement in the understanding of the role of SLC4A1l in cancer. The further elucidation of underlying mechanism could be useful for the development of a novel SLC4A11-targeted strategy for GC treatment.

\section{Acknowledgments}

This study was supported by grants of the 13th Five Year Key Talent's Subsidy Project in Science and Education of Jiangsu Province (ZDRCA2016051) and Jiangsu Provincial Commission of Health and Family Planning (LGY2016038). We thank James P. Mahaffey, PhD, from Liwen Bianji, Edanz Editing China (www.liwenbianji.cn/ac), for editing the English text of a draft of this manuscript.

\section{References}

[1] Bray F, Ferlay J, Soerjomataram I, et al. Global cancer statistics 2018: GLOBOCAN estimates of incidence and mortality worldwide for 36 cancers in 185 countries[J]. CA Cancer J Clin, 2018, 68 (6): 394-424.

[2] Liu D, Xia P, Diao D, et al. MiRNA-429 suppresses the growth of gastric cancer cells in vitro[J]. J Biomed Res, 2012, 26 (5): 389-93.

[3] Dai C, Zhao L, Fan Z. An unusual esophageal dysphagia under gastroscopy and endoscopic ultrasonography $[\mathrm{J}]$. Gastroenterology, 2017, 152 (4): 699-701.

[4] Sun W, Yan L. Gastric cancer: current and evolving treatment landscape[J]. Chin J Cancer, 2016, 35 (1): 83.
[5] Yao Z, Fenoglio S, Gao DC, et al. TGF-beta IL-6 axis mediates selective and adaptive mechanisms of resistance to molecular targeted therapy in lung cancer[J]. Proc Natl Acad Sci U S A, 2010, 107 (35): 15535-40.

[6] Gao P, Sun X, Chen X, et al. Secretable chaperone Grp170 enhances therapeutic activity of a novel tumor suppressor, MDA-7/IL-24[J]. Cancer Res, 2008, 68 (10): 3890-8.

[7] Aalkjaer C, Boedtkjer E, Choi I, et al. Cation-coupled bicarbonate transporters[J]. Compr Physiol, 2014, 4 (4): 1605-37.

[8] Thornell IM, Bevensee MO. Regulators of Slc4 bicarbonate transporter activity[J]. Front Physiol, 2015, 6:166.

[9] Zhang W, Ogando D G, Bonanno J A, et al. Human SLC4A11 is a novel $\mathrm{NH}_{3} / \mathrm{H}^{+}$co-transporter[J]. $J$ Biol Chem, 2015, 290 (27): 16894-905.

[10] Nehrke $\mathrm{K} . \mathrm{H}(\mathrm{OH}), \mathrm{H}(\mathrm{OH}), \mathrm{H}(\mathrm{OH})$ : a holiday perspective. Focus on "Mouse Slc4all expressed in Xenopus oocytes is an ideally selective $\mathrm{H}^{+} / \mathrm{OH}^{-}$conductance pathway that is stimulated by rises in intracellular and extracellular $\mathrm{pH} "[\mathrm{~J}]$. Am J Physiol Cell Physiol, 2016, 311 (6): C942-C944.

[11] Parker MD, Ourmozdi EP, Tanner M J. Human BTR1, a new bicarbonate transporter superfamily member and human AE4 from kidney[J]. Biochem Biophys Res Commun, 2001, 282 (5): 1103-9.

[12] Roy S, Praneetha DC, Vendra VP. Mutations in the corneal endothelial dystrophy-associated gene SLC4A11 render the cells more vulnerable to oxidative insults [J]. Cornea, 2015, 34 (6): 668-74.

[13] Loganathan SK, Schneider HP, Morgan PE, et al. Functional assessment of SLC4A11, an integral membrane protein mutated in corneal dystrophies[J]. Am J Physiol Cell Physiol, 2016, 311 (5): C735-48.

[14] Vithana EN, Morgan P, Sundaresan P, et al. Mutations in sodium-borate cotransporter SLC4A11 cause recessive congenital hereditary endothelial dystrophy (CHED2) [J]. Nat Genet, 2006. 38 (7): 755-7.

[15] Qin L, Li T, Liu Y. High SLC4A11 expression is an independent predictor for poor overall survival in grade 3/4 serous ovarian cancer[J]. PLoS One, 2017, 12 (11): $\mathrm{e} 0187385$.

[16] Jiang C, Zhu J, Zhou P, et al. Overexpression of FIBCD1 is predictive of poor prognosis in gastric cancer[J]. Am J Clin Pathol, 2018, 149 (6): 474-83.

[17] Li J, Jin Q, Huang F, et al. Effects of Rab27A and Rab27B on invasion, proliferation, apoptosis, and chemoresistance in human pancreatic cancer cells[J]. Pancreas, 2017, 46 (9): 1173-9.

[18] Schneider CA, Rasband WS, Eliceiri KW. NIH image to ImageJ: 25 years of image analysis[J]. Nat Methods, 2012, 9 (7): 671-5.

[19] Sun P, Cao X, Liu Y, et al. Eg5 high expression predicts dismal prognosis in epithelial ovarian cancer[J]. AsiaPacific Journal of Blood Types and Genes, 2019, 2 (3): 145-51.

[20] Zhai X, Wang Y, Chen P, et al. High CCNB2 expression correlates with poor prognosis in hepatocellular carcinoma[J]. Asia-Pacific Journal of Blood Types and 
Genes, 2019, 3 (1): 55-62.

[21] Huang J, Mei H, Tang Z, et al. Triple-amiRNA VEGFRs inhibition in pancreatic cancer improves the efficacy of chemotherapy through EMT regulation[J]. J Control Release, 2017, 245: 1-14.

[22] Detre S, Saclani Jotti G, Dowsett M. A "quickscore" method for immunohistochemical semiquantitation: validation for oestrogen receptor in breast carcinomas[J]. J Clin Pathol, 1995, 48 (9): 876-8.

[23] Romero MF, Chen AP, Parker MD, et al. The SLC4 family of bicarbonate $(\mathrm{HCO}(3)(-))$ transporters[J]. Mol Aspects Med, 2013, 34 (2-3): 159-82.

[24] Hu MY, Yan JJ, Petersen I, et al. A SLC4 family bicarbonate transporter is critical for intracellular $\mathrm{pH}$ regulation and biomineralization in sea urchin embryos[J]. Elife, 2018, 7: e36600.

[25] Alper SL. Molecular physiology and genetics of $\mathrm{Na}^{+}$-independent SLC4 anion exchangers[J]. J Exp Biol, 2009, 212 (Pt 11): 1672-83.

[26] Kao L, Azimov R, Abuladze N, et al. Human SLC4A11C functions as a DIDS-stimulatable $\mathrm{H}(+)(\mathrm{OH}(-))$ permeation pathway: partial correction of $\mathrm{R} 109 \mathrm{H}$ mutant transport[J]. Am J Physiol Cell Physiol, 2015, 308 (2): C176-88.

[27] Kodaganur SG, Kapoor S, Veerappa AM, et al. Mutation analysis of the SLC4A11 gene in Indian families with congenital hereditary endothelial dystrophy 2 and a review of the literature[J]. Mol Vis, 2013, 19: 1694-706.

[28] Hand CK, McGuire M, Parfrey NA, et al. Homozygous SLC4A11 mutation in a large Irish CHED2 pedigree[J]. Ophthalmic Genet, 2017, 38 (2): 148-51.

[29] Kim JH, Ko JM, Tchah H. Fuchs endothelial corneal dystrophy in a heterozygous carrier of congenital hereditary endothelial dystrophy type 2 with a novel mutation in
SLC4A11[J]. Ophthalmic Genet, 2015, 36 (3): 284-6.

[30] Loganathan SK, Casey JR. Corneal dystrophy-causing SLC4A11 mutants: suitability for folding-correction therapy[J]. Hum Mutat, 2014, 35 (9): 1082-91.

[31] Han SB, Ang HP, Poh R, et al. Mice with a targeted disruption of Slc4all model the progressive corneal changes of congenital hereditary endothelial dystrophy[J]. Invest Ophthalmol Vis Sci, 2013, 54 (9): 6179-89.

[32] Guha S, Chaurasia S, Ramachandran C, et al. SLC4A11 depletion impairs NRF2 mediated antioxidant signaling and increases reactive oxygen species in human corneal endothelial cells during oxidative stress[J]. Sci Rep, 2017, 7 (1): 4074 .

[33] Liu J, Seet LF, Koh LW, et al. Depletion of SLC4A11 causes cell death by apoptosis in an immortalized human corneal endothelial cell line[J]. Invest Ophthalmol Vis Sci, 2012, 53 (7): 3270-9.

[34] McIntyre A, Hulikova A, Ledaki I, et al. Disrupting hypoxia-induced bicarbonate transport acidifies tumor cells and suppresses tumor growth[J]. Cancer Res, 2016, 76 (13): 3744-55.

[35] Parks SK, Pouyssegur J. The $\mathrm{Na}(+) / \mathrm{HCO}_{3}(-)$ co-transporter SLC4A4 plays a role in growth and migration of colon and breast cancer cells[J]. J Cell Physiol, 2015, 230 (8): 1954-63.

[36] Badior KE, Alka K, Casey JR. SLC4A11 Three-dimensional homology model rationalizes corneal dystrophycausing mutations[J]. Hum Mutat, 2017, 38 (3): 279-88.

[37] Loganathan SK, Lukowski CM, Casey J R. The cytoplasmic domain is essential for transport function of the integral membrane transport protein SLC4A11[J]. Am J Physiol Cell Physiol, 2016, 310 (2): C161-74.

Received 24 July 2019, Revised 17 December 2019, Accepted 13 January 2020 